\title{
Neo-adjuvant chemo-radiation therapy in T4 resectable oral cavity cancer
}

\author{
Cheemala Sushmitha ${ }^{1 * *}$, Geeta S Narayanan ${ }^{1 *}$, Ganesh MS $^{2 *}$, Vasu Reddy ${ }^{2}$, Bhaskar Vishwanathan ${ }^{1}$, Sanjeet Kumar Mandal ${ }^{1}$, Sowmya \\ Narayanan $^{3}$, Shadhidhar V Karupaiah ${ }^{4}$ and Manjunath $^{4}$ \\ ${ }^{1}$ Department of radiation oncology, Vydehi institute of medical sciences and research Centre, Bengaluru, India \\ ${ }^{2}$ Department of Surgical oncology, Vydehi institute of medical sciences and research Centre, Bengaluru, India \\ ${ }^{3}$ Department of Radiation Physics, Vydehi institute of medical sciences and research Centre, Bengaluru, India \\ ${ }^{4}$ Department of Radiation Physics, Vydehi institute of medical sciences and research Centre, Bengaluru, India \\ ${ }^{\#}$ Authors equally contributed
}

\begin{abstract}
Purpose: Primary objective is to evaluate clinical and pathological response at both primary and neck and margin status and postoperative morbidity in T4 resectable oral cavity cancer patients treated with neo adjuvant chemo-radiotherapy.

Methods: 30 patients, presenting with T4 resectable oral cavity cancers were randomly assigned to Arm A (Neo-adjuvant chemo-radiotherapy followed Surgery was performed after 2 to 6 weeks) and Arm B (Definitive Surgery followed by adjuvant radiotherapy with or without chemotherapy).

Results: In our study, out of fifteen patients in neo-adjuvant chemo-radiation, who underwent surgery in which $7(46.7 \%)$ had pathological complete response with no residual disease, $6(40 \%)$ with partial response with negative margins and negative nodes. No significant difference observed in margin positivity compared with standard arm ( $\mathrm{p}=0.88)$. Postoperative morbidity was comparable. Some of the patients could undergo limited resections.

Conclusion: Our study concludes that neo adjuvant chemo-radiotherapy followed by surgery is feasible with good pathological complete responses. Since response to chemo radiotherapy is an important prognostic factor, results may translate in better disease-free survival in the future.
\end{abstract}

\section{Introduction}

Although progress has been achieved in radical surgical resection with reconstruction and use of postoperative radiotherapy/chemoradiotherapy, the 5-year survival rate has not improved substantially in recent years, remaining at $50 \%$ to $60 \%[1,2]$. Statistics of surgical departments indicate most of them recur regionally or distantly in the first two years in spite of initial surgery and radiotherapy [3].

To improve local-regional control and to allow better functional and cosmetic results after surgery, neo-adjuvant protocols have been developed during the last decade implementing radio-chemotherapy prior to selective surgery. Currently there is no well-established protocol for neo-adjuvant chemo-radiotherapy for resectable oral cavity cancers. Hence this study is undertaken. We have chosen resectable $\mathrm{T} 4$ oral cancers because of high incidence of close margins (especially in tumors of retromolar trigone and upper bucco-gingival region extending posteriorly) reported higher local recurrence rates in Indian population.

\section{Objectives}

1. To evaluate clinical and pathological response at both primary and neck and margin status in T4 resectable oral cavity cancer patients treated with neo adjuvant chemo-radiotherapy. 2.To evaluate postoperative morbidity.

\section{Methods}

The source of data for the study were patients presenting to the department of oncology, Vydehi Institute of Medical Sciences, Bangalore with stage T4 resectable oral cavity cancers. Patients recruited from Jan 2014 to June 2015.

The sample size chosen was 30 . This was estimated based on data obtained with the historical studies.

ARM A: Neo adjuvant chemo radiotherapy.

ARM B: Surgery and adjuvant chemo and/or radiotherapy.

Inclusion Criteria: 1 . Age 20 to 65 years, 2. Performance Status-0-2 (ECOG Criteria). 3. Primary-T4 resectable invasive squamous cell carcinoma of the oral cavity (According to AJCC $7^{\text {th }}$ edition) 4 . Neck -N0, N1, N2b and N2c (Nodal size less than $3 \mathrm{~cm}$, mobile).

*Correspondence to: Cheemala Sushmitha, Assistant Professor, Vydehi Institute of Medical Sciences and Research Centre, No. 82, EPIP area Nallurahalli, Bengaluru, India, Tel: 9742912997; E-mail: Sushmithadr@gmail.com

Key words: neo adjuvant chemo-radiation, resectable, T4, oral cavity

Received: May 18, 2019; Accepted: June 24, 2019; Published: June 27, 2019 
Exclusion Criteria: 1. Metastatic disease, 2. Performance status more than 2, 3. Previous irradiation to head and neck area, 4. Patients with fixed nodes and nodal size more than $3 \mathrm{~cm}$.

Informed and written consent of the patient taken.

\section{Treatment}

Out of 30 patients, presenting with stage IV oral cavity cancers 15 patients were randomly assigned to neo-adjuvant chemo radiation (Arm A). Radiotherapy was delivered to a dose of 4600 cGy in 23 fractions, 200cGy per fraction, 5 fractions per week to primary and neck, using 3-dimensional conformal radiation therapy (3DCRT) with $6 \mathrm{MV}$ photons. Chemotherapy drug was Inj. Cisplatin $\left(70 \mathrm{mg} / \mathrm{m}^{2}\right)$ which was delivered concurrently with radiation therapy on day 1 and day 21. Surgery was performed after 4-6 weeks and 15 patients in (Arm B) definitive Surgery followed by adjuvant radiotherapy with or without chemotherapy (Positive margin/Extra nodal extension).Radiation was delivered to a dose of $6000 \mathrm{cGy}$ in 30 fractions, 200cGy per fraction, 5 fractions per week to primary and neck, using 3 dimensional conformal radiation therapy (3DCRT) with $6 \mathrm{MV}$ photons and Chemotherapy was delivered with Inj.Cisplatin $\left(70 \mathrm{mg} / \mathrm{m}^{2}\right)$ concurrently with radiation therapy on day 1 and day 21 . All patients were intravenously pre medicated with dexamethasone, ranitidine and standard antiemetic agents.

\section{During treatment}

The patients were explained about the care of irradiated site, precautions, and diet modifications. The weight of the patient was checked on a weekly basis. Acute reactions were recorded using RTOG Acute Reaction Morbidity Criteria. The patients were managed according to the toxicity profile.

\section{Response assessment}

At 4 weeks patients were assessed by clinical and radiological examination with contrast CT of the Head and neck. Responses were categorized according to RECIST criteria 1.1.

After surgery, post op histo-pathological response was assessed at primary and nodes, any other positive risk features were documented. Adjuvant radiation/chemotherapy was given ONLY for positive margins/positive nodes with ECE.

\section{Follow up}

Patients were assessed for post treatment morbidity and were followed up at $6 \mathrm{wks}, 3$ months, 6 months.

\section{Statistical analysis}

Our study is a randomized, prospective interventional study.

\section{Statistical Methods:}

Data were analyzed using SPSS version 20 for windows. Frequency distribution of response and categorical variables were determined. Chi square test for proportions to compare differences between neoadjuvant chemoradiation arm and standard treatment arm for site, stage, surgery type, positive margins, and postoperative morbidity was determined. $\mathrm{P}$ value $<0.05$ was considered statistically significant.

\section{Results}

All the patients had T4a resectable oral cavity cancers, Sub sites were comparable in both arms, but the nodal status was higher in the neo-adjuvant arm as shown in the Table 1 . Response assessment was done at 4 weeks of post CTRT by clinical and radiological examination which showed $70.6 \%$ partial response and $17.6 \%$ complete response (Figures 1 and 2). Fifteen patients underwent surgery within 4-9 weeks. Thirteen patients underwent composite resection and 2 patients wide local excision (Figure 3). Histologically $46.7 \%$ patients shows complete response at primary and $100 \%$ at nodes (Figure 4 ). There was one treatment related mortality in Neo-adjuvant chemo-radiation arm. Margin status andmorbidity was comparable in both the arms (Figures 5 and 6).

\section{Discussion}

To our best knowledge, this is the first prospective study in India to compare Neo-adjuvant chemo-radiotherapy (CRT) and surgery followed by radiotherapy with/without chemotherapy in the treatment of resectable $\mathrm{T} 4$ oral cavity cancer.

Although surgery is the mainstream of therapy in oral cavity Squamous cell carcinoma,most patients in stage IV with bone and deep

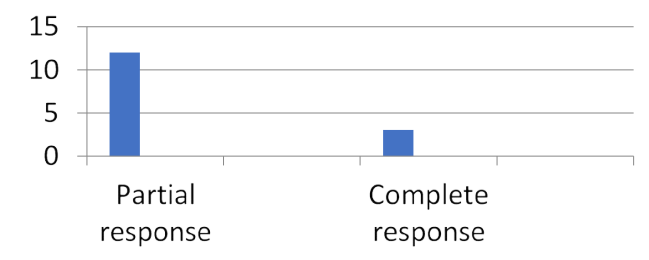

Figure 1. Bar diagram showing clinical and radiological response -Primary

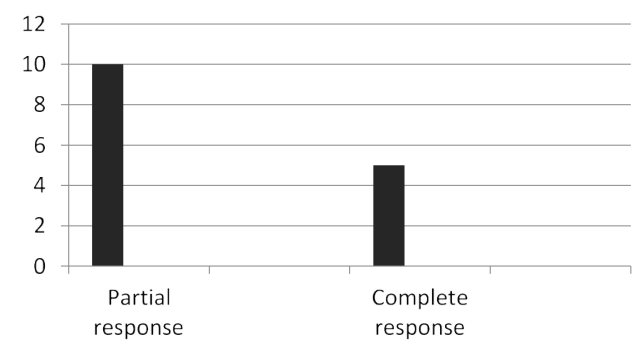

Figure 2. Bar diagram showing clinical and radiological response - Node

Table 1. Patient and tumour characteristics

\begin{tabular}{|c|c|c|c|}
\hline & $\begin{array}{c}\text { NEOADJUVANT } \\
\text { ARM }\end{array}$ & $\begin{array}{c}\text { STANDARD } \\
\text { ARM }\end{array}$ & P Value \\
\hline \multicolumn{4}{|l|}{ Age } \\
\hline $20-39$ & 2 & 1 & \\
\hline $40-59$ & 10 & 12 & \\
\hline $60-65$ & 3 & 2 & \\
\hline \multicolumn{4}{|l|}{ Sex } \\
\hline Male & $6(40 \%)$ & $7(43 \%)$ & 0.74 \\
\hline Female & $9(60 \%)$ & $8(53 \%)$ & \\
\hline \multicolumn{4}{|l|}{ Sub site } \\
\hline $\begin{array}{l}\text { Buccal mucosa extending to } \\
\text { Retromolar trigone }\end{array}$ & $9(60 \%)$ & $10(66 \%)$ & 0.4 \\
\hline Buccal mucosa & $1(10 \%)$ & $2(13 \%)$ & \\
\hline Lower alveolus & $2(13 \%)$ & $1(10 \%)$ & \\
\hline Retromolar trigone & $3(20 \%)$ & $1(10 \%)$ & \\
\hline Tongue & - & $1(15 \%)$ & \\
\hline Upper Alveolus & - & - & \\
\hline \multicolumn{4}{|l|}{ N STATUS } \\
\hline N0 & 0 & $8(53 \%)$ & 0.001 \\
\hline N1 & $12(80 \%)$ & $5(33 \%)$ & \\
\hline $\mathrm{N} 2$ & $3(20 \%)$ & $2(13 \%)$ & \\
\hline
\end{tabular}




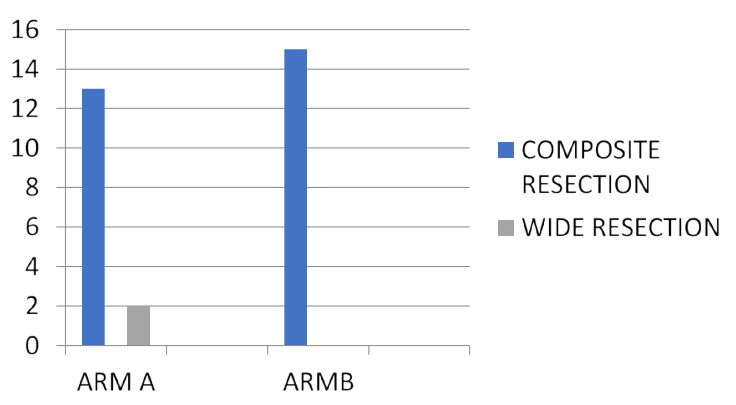

Figure 3. Bar diagram showing type of surgery done

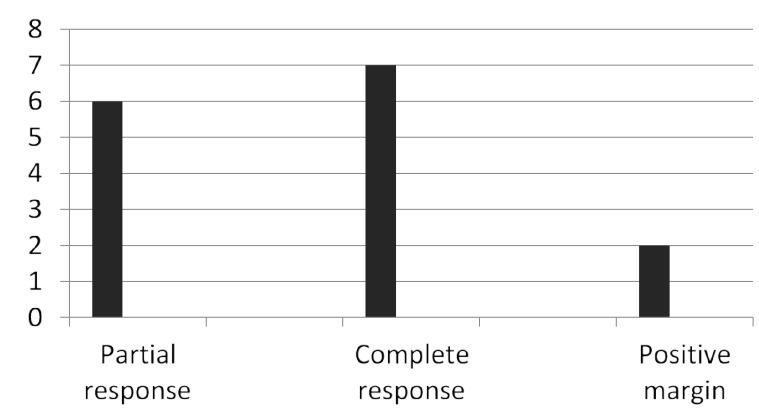

Figure 4. Bar diagram showing pathological response: ARM A

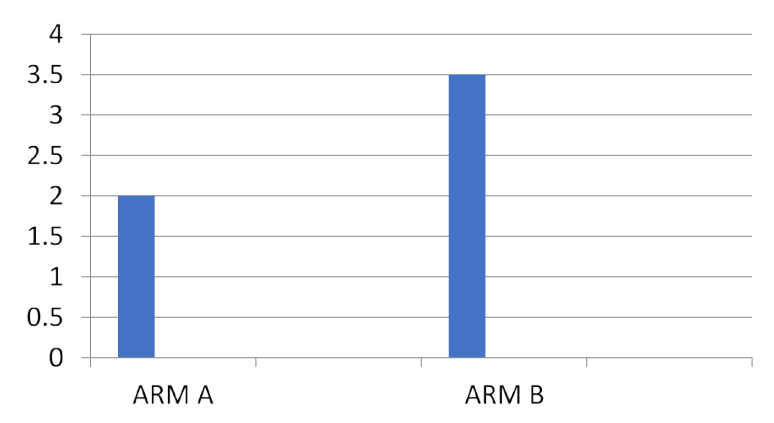

Figure 5. Bar diagram positive margins status

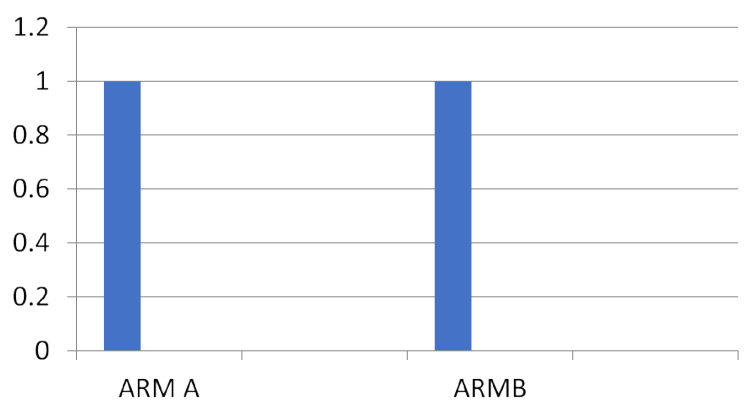

Figure 6. Bar diagram showing postopmorbidity

muscles of tongue end up with severe morbidity and are more likely to have positive margins. Adjuvant chemo-radiotherapy is associated with acute mucositis, dysphagia, nausea, vomiting, hoarness and long term complications such as esophageal stenosis, hypothyroidism, osteoradionecrosis, xerostomia and subcutaneous fibrosis [4].

Sum studies on preoperative radiation in locally advanced head and neck cancer was reportedly associated with more toxicity than postoperative RT by the results of RTOG multicenter phase 3 trial 7303 [5-7].
Studies on preoperative chemo-radiation followed by surgery showed improved disease free survival and overall survival and acceptable toxicity [8-14].

Cisplatin has been studied most extensively, achieving significantly greater regression of experimental tumors when combined with radiation than did either technique alone [15,16], with partial and complete tumor responses in many patients with non resectable squamous cell carcinoma of head and neck $[17,18]$.

Addition of cisplatin to preoperative radiotherapy did not enhance OS, but significantly improved 5-year DMFS and also borderline significance in PFS when compared to preoperative radiotherapy alone [19].

One of the most significant findings of our study was the large number of pathologically complete responses at primary (46.7\%) and node $(100 \%)$ after neo-adjuvant CT/RT. The drawback here was that these nodes were detected on clinical and radiological examination and were not proven with histopathology before starting neo-adjuvant chemo radiation. Also the inclusion criteria was to include nodes up to $3 \mathrm{~cm}$.But considering the fact that $30 \%$ of the clinically N0 nodes show microscopic disease, neo-adjuvant chemo radiation has definitely helped to treat these cases.

In the literature, studies have reported that response to neoadjuvant chemotherapy was the most important prognostic factor $[9,10]$. Our study has shown high pathological complete responses, this may translate in to better disease free survival and overall survival though long term follow up would be required to document these findings. Adjuvant radiation was needed in only one patient with positive margin.

Our results showed that neo-adjuvant CT/RT followed by surgery was well tolerated with acceptable toxicity similar to previous study [9]. During neo-adjuvant CT/RT, mucositis of grade 3 was observed in $9(45 \%)$ patients and were managed symptomatically. Two patients had radiation treatment interruption of two days. Only one patient developed grade 3 neutropenia. One patient in neo-adjuvant arm died in the postoperative period due to septicemia. No other major complications were observed. Though statistically not significant, two patients could undergo wide local excision after neo-adjuvant chemo radiation. The use neo-adjuvant chemo-radiation in achieving limited resections needs to be addressed further. Postoperative morbidity was comparable with standard arm. Only one patient in the neo-adjuvant chemo-radiation arm developed fistula. One patient in standard arm had graft failure. Long term follow up will be required to further assess the effectiveness of neo-adjuvant therapy.

\section{Conclusion}

1. A higher pathological complete response could be achieved at both primary and selected neck nodal disease by neo-adjuvant chemo-radiotherapy in patients with resectable T4a squamous cell carcinoma oral cancers.

2. The higher pathological response could be a predictor of better disease-free survival and overall survival.

3. The neo-adjuvant chemo-radiation did not increase toxicity and post op morbidity.

\section{References}

1. Neville BW, Day TA (2002) Oral cancer and precancerous lesions. CA Cancer J Clin 52: 195-215. [Crossref] 
2. Parkin DM, Bray F, Ferlay J (2002) Global cancer statistics. CA Cancer J Clin 55: 74-108. [Crossref]

3. Oliver RJ, Clakson JE, Conway DI (2007) Interventions for the treatment of oral and oropharyngealcancers: surgicaltreatment. Cochrane Database System Rev (4): CD006205.

4. Cmelac A, Arneson K, Chau NG (2013) Locally advanced head and neck cancer. Am Soc Oncol Educ Book pp: 237-44.

5. Kramer S, Gelber RD, Snow JB, Marcial VA, Lowry LD, et al. (1987) Combined radiation therapy and surgery in the management of advanced head and neck cancer: final report of study 73-03 of the Radiation Therapy Oncology Group. Head Neck Surg 10: 19-30. [Crossref]

6. Tupchong L, Scott CB, Blitzer PH, Marcial VA, Lowry LD, et al. (1991) Randomized study of preoperative versus postoperative radiation therapy in advanced head and neck carcinoma: long-term follow-up of RTOG study 73-03. Int J Radiat Oncol Biol Phys 20: 21-28.

7. Vandenbrouck C, Sancho H, Le Fur R, Richard JM, Cachin Y (1977) Results of randomized clinical trial of preoperative irradiation versus postoperative in treatment of tumors of the hypopharynx. Cancer 39: 1445-1449.

8. Gus J Slotman, Charles H, Doolittl e, Arvin S, Glicksman, et al (1992) Preoperative combined chemotherapy and radiotherapy plus radical surgery in advanced Head Neck cancer.Five -year results with impressive complete response rates and high survival. Cancer 69: 2736-2743.

9. Klug C, Wultz A, Kermer C (2005) Preoperative radiochemotherapy and radical resection for stages II to IV oral and oropharyngeal cancer: grade of regression as crucial prognostic factor. International Journal of Oral and Maxillofacial Surgery 34: 262-267.

10. Klug C, Wultz A, Kermer C (2005) Preoperative radiochemotherapy and radical resection for stages II-IV oral and oropharyngeal cancer: outcome of 222 patients. International Journal of Oral and Maxillofacial Surgery 34: 143-148.
11. Kolja F, Michael E, Katia L (2008) Neoadjuvant concurrent radiochemotherapy followed by surgery in advanced oral squamous cell carcinoma (OSCC): A retrospective analysis of 207 patients. Oral Oncology 44: 116-123.

12. Thomas M, Mario K, Stefen W (2011) Low-Dose Preoperative Chemoradiation Therapy Compared with Surgery Alone with or Without Postoperative Radiotherapy in Patients with Head and Neck Carcinoma. Annals of Surgical Oncology 18: 2739-2747.

13. Kreppel M, Dreiseidler T, Rothamel D (2013) The role of clinical versus histopathological staging in patients with advanced oral squamous cell carcinoma treated with neoadjuvant radiochemotherapy followed by radical surgery. $J$ Craniomaxillofac Surg 41: 22- 27. [Crossref]

14. Kreppel M, Hans TE, Christian B (2012) Preoperative vs. postoperative radiochemotherapy in patients with N2 squamous cell carcinoma of the oral cavity. Oral Oncology 48: 1019-1124. [Crossref]

15. Douple EB, Eaton WL, Tulloh ME (1979) Skin radiosensitization studies using combined cis-dichlorodiammineplatinum (II) and radia- tion. Int J Radial Oneal Biol Phys 5: 1383-1385.

16. Soloway MS, Morris CR, Sudderth B (1979) Radiation therapy and cisdiamminedichloroplatinum (II) in transplantable and pri- mary murine bladder cancer. Int J Radial Oneal Biol Phys 5: 1355-1360.

17. Leipzig B (1983) Cisplatin sensitization to radiotherapy of squamous cell carcinoma of the head and neck. Am J Surg 146: 462- 465.

18. Leipzig B, Wetmore SJ, Putzeys MD (1985) Cisplatin potentiation of radiotherapy. Arch Otolaryngol Head Neck Surg 111: 114-118.

19. Phase III randomized trial of preoperative concurrent chemoradiotherapy versus preoperative radiotherapy for patients with locally advanced head and neck squamous cell carcinoma. Oncotarget 8: 44842-44850

Copyright: (02019 Sushmitha C. This is an open-access article distributed under the terms of the Creative Commons Attribution License, which permits unrestricted use, distribution, and reproduction in any medium, provided the original author and source are credited. 